\title{
Heterotopic Ossification: Clinical Features, Basic Researches, and Mechanical Stimulations
}

\author{
Yili Xu ${ }^{1}$, Mei Huang ${ }^{1}$, Wenzhen $\mathrm{He}^{1}$, Chen $\mathrm{He}^{1}$, Kaixuan Chen ${ }^{1}$, Jing Hou ${ }^{1}$, Min Huang ${ }^{1}$, \\ Yurui Jiao ${ }^{1}$, Ran Liu ${ }^{1}$, Nanyu Zou ${ }^{1}$, Ling Liu ${ }^{1}$ and Changjun $L^{1}{ }^{1,2,3 *}$ \\ ${ }^{1}$ Department of Endocrinology, Endocrinology Research Center, The Xiangya Hospital of Central South University, Changsha, \\ China, ${ }^{2}$ National Clinical Research Center for Geriatric Disorders (Xiangya Hospital), Changsha, China, ${ }^{3}$ Key Laboratory of Organ \\ Injury, Aging and Regenerative Medicine of Hunan Province, Changsha, China
}

Heterotopic ossification $(\mathrm{HO})$ is defined as the occurrence of extraskeletal bone in soft tissue. Although this pathological osteogenesis process involves the participation of osteoblasts and osteoclasts during the formation of bone structures, it differs from normal physiological osteogenesis in many features. In this article, the primary characteristics of heterotopic ossification are reviewed from both clinical and basic research perspectives, with a special highlight on the influence of mechanics on

OPEN ACCESS

Edited by:

Airong Qian,

Northwestern Polytechnical

University, China

Reviewed by:

Chong Yin,

Affiliated Hospital of North Sichuan

Medical College, China

*Correspondence:

Changjun $\mathrm{Li}$

lichangjun@csu.edu.cn

Specialty section:

This article was submitted to Morphogenesis and Patterning,

a section of the journal

Frontiers in Cell and Developmental

Biology

Received: 05 September 2021 Accepted: 03 January 2022

Published: 25 January 2022

Citation:

Xu Y, Huang M, He W, He C, Chen $K$, Hou J, Huang M, Jiao Y, Liu R, Zou N,

Liu L and Li C (2022) Heterotopic

Ossification: Clinical Features, Basic

Researches, and

Mechanical Stimulations.

Front. Cell Dev. Biol. 10:770931.

doi: 10.3389/fcell.2022.770931 heterotopic ossification, which serves an important role in the prophylaxis and treatment of $\mathrm{HO}$.

Keywords: heterotopic ossification, mechanical loading, bone, stem cell fate, bone formation

\section{INTRODUCTION}

Heterotopic ossification ( $\mathrm{HO}$ ) is a complicated pathologic process causing the formation of extraskeletal bone in soft tissues, such as muscle, peri-articulations, ligaments, and tendons. It is commonly recognized as a complication after trauma, surgery, blast, spinal cord injury, and other stress damages (Shimono et al., 2011; Regard et al., 2013; Ranganathan et al., 2015; Wang et al., 2016). Heterotopic ossification was first labeled as "paraosteoarthropathy" by French physicians Dejerne and Ceillier, being a consequence of traumatic paraplegia of patients during World War I, and was further observed among soldiers returning from Iraq and Afghanistan (Naraghi et al., 1996; Forsberg et al., 2009; Potter et al., 2010; Forsberg et al., 2014). In severe cases, complete bony ankylosis as a result of $\mathrm{HO}$ is quite common, and more than $20 \%$ of patients appear overt dysfunction in soft-tissue, joint, or suffer from chronic pain; The HO morbidity of patients with traumatic brain injury almost reach 50\% (Vanden Bossche and Vanderstraeten, 2005; Balboni et al., 2006; Zhang et al., 2014; Xu et al., 2018).

Inquiry about the underlying mechanism, such as cellular and mechanical processes, and earlier diagnoses as well as more effective treatments, is the hotspot of current research. Scientists analyze the proteomic biomarkers to identify early diagnostic indexes based on high-throughput mass spectrometry and antibody arrays; Doctors seek to develop efficacious prophylactic management and specific treatments via physical therapy, pharmaceutical intervention, operation, and radiation

Abbreviations: AHO, Albright's hereditary osteodystrophy; BMP, bone morphogenetic protein; BNB, blood-nerve barrier; FOP, fibrodysplasia ossificans progressive; HIFs, Hypoxia-inducible factors; HO, Heterotopic ossification; MSPCs, mesenchymal stromal/progenitor cells; NSAIDs, Nonsteroidal Antiinflammatory Drugs; POH, progressive osseous heteroplasia TDPCs, tendon-derived progenitor cells; TMJA, temporomandibular joint ankylosis; TMJ, temporomandibular joint. 
(Yuan et al., 2009; Coons and Godleski, 2013; Cheng et al., 2017; Gomez-Puerto et al., 2019; Botman et al., 2020). Moreover, patients with a high incidence of traumatic heterotopic ossification, such as fractures and hip joint arthroplasty, need to undergo prolonged postoperative immobilization or early rehabilitation exercises. Post-traumatic motion and mechanical loading are closely related to the occurrence of heterotopic ossification. The role of passive motion rehabilitative therapy after trauma, fracture, or invasive surgery for heterotopic ossification is still controversial. In this review, we elaborate on the clinical features and the fundamental biological mechanisms of $\mathrm{HO}$, and for the first time summarize the separate influences of mechanical stimulations on $\mathrm{HO}$ based on up-to-date researches.

\section{CLINICAL FEATURES OF HETEROTOPIC OSSIFICATION}

\section{Epidemiology}

$\mathrm{HO}$ is often divided into three categories: traumatic, neurogenic, and genetic. The prevalence of traumatic-induced $\mathrm{HO}$ following burn injury has been reported to range from 0.2 to $4 \%$, and up to $90 \%$ following the total hip joint arthroplasty or acetabular fractures (Cipriano et al., 2009; Maender et al., 2010; Rath et al., 2013; Medina et al., 2014; Medina et al., 2015). The predilection age of trauma-induced $\mathrm{HO}$ is 20-40 years old. Approximately half of $\mathrm{HO}$ occurs at this age. However, the other half of the $\mathrm{HO}$ could present dispersedly from infancy to late adulthood (Ackerman, 1958; Elmas and Shrestha, 2017; Xu et al., 2017; Meyers et al., 2019; Kaliya-Perumal et al., 2020). The morbidity of heterotopic ossification following central neurologic injury has been calculated to range from 10 to $53 \%$ (Teasell et al., 2010). Most studies regard traumatic brain-injured patients and spinal cord injured patients as the same category. And the prevalence of genetic $\mathrm{HO}$, including fibrodysplasia ossificans progressiva (FOP), progressive osseous heteroplasia ( $\mathrm{POH}$ ), and Albright's hereditary osteodystrophy (AHO) (Shore and Kaplan, 2010), is extremely rare, affecting 1 in 2,000,000 people (Baujat et al., 2017). However, genetic $\mathrm{HO}$ is consensually regarded as the most severe $\mathrm{HO}$ disease in humans (Qi et al., 2017; Kaliya-Perumal et al., 2020). Male sex, the amount, and the type of motion could also raise the risk of $\mathrm{HO}$. Men are slightly more vulnerable to $\mathrm{HO}$ with a sex ratio of 3:2 (Meyers et al., 2019), perhaps due to the various muscle mass, differential level of physical activity, repetitive mechanical stress working as "microtrauma", and distinct hormonal signaling pathways affecting osteogenesis (Ranganathan et al., 2015; Ko et al., 2016; Malca et al., 2018; Dowdell et al., 2020; Rüdiger et al., 2020).

\section{Clinical Presentation}

The typical clinical features of $\mathrm{HO}$ include the limited range of motion around the involved joint, complete bony ankylosis in severe cases, and deformity in the cervical spine, elbow, shoulder, fingers, jaw exostosis, or temporomandibular joint ankylosis (TMJA) (Zhao et al., 2020). HO could occur almost anywhere in the body, as long as it is associated with the periosteum. Typically, HO initiates away from the periosteum, and then fuse to the periosteum as a secondary feature (Meyers et al., 2019). But it is rare to observe $\mathrm{HO}$ in some anatomic tissues, such as the viscera or the diaphragm. This might be due to the lack of pluripotent stem cells in these sites or because these sites are not mechanically stimulated as often as the peri-articular areas prone to heterotopic ossification. Moreover, $\mathrm{HO}$ can only be detected as an asymptomatic finding on a radiograph. It is quite challenging to identify the potential biomarkers for early disease detection and monitoring, let alone the symptom present with complications that usually confound diagnosis (Crowgey et al., 2018). There are several ways to classify HO diseases. Four levels of classification for $\mathrm{HO}$ around the hip were set by Brooker to indicate the severity (Brooker et al., 1973). The Hastings and Graham classification system classifies $\mathrm{HO}$ at the elbow into three grades based on clinical and radiographic data (Hastings and Graham, 1994).

The presentations of genetic $\mathrm{HO}$ are more serious than traumatic-induced HO. Almost all FOP patients reported to date were caused by Acvr1 mutation, and showing abnormality early. Acvr1 gene locates on chromosome 2 (2q23-24) and encodes a bone morphogenetic protein (BMP) type 1 receptor, which is generally considered to be the major regulator in $\mathrm{HO}$ pathophysiology (Wang et al., 2016; Haupt et al., 2019; Meyers et al., 2019; Pearson et al., 2019; Stanley et al., 2019; Botman et al., 2020; Kaliya-Perumal et al., 2020). Acvr1 mutation results in abnormally enhanced sensitivity of this receptor to BMPs, allowing for overexcitation of the BMP/SMAD pathway and heterotopic ossification. The typical feature of FOP is multiple skeletal deformities, involving fingers, toes, and cervical spine, and eventually resulting in pain, movement, and function limitation. $\mathrm{POH}$ is a genetic $\mathrm{HO}$ caused by inactivating mutations in the GNAS1 gene, which result in decreased expression or function of the alpha subunit of the stimulatory G protein (Gsa) of adenylyl cyclase (Zhang et al., 2018). POH is characterized by intramembranous and cutaneous ossification, and could occur on the ear or fingers as an atypical phenotype (Kaplan et al., 1994; Zhang et al., 2018).

However, HO may be alleviated by physical intervention for traumatic-induced patients such as immobilization or Long-term bedridden. Doctors routinely use immobilization for extremity trauma patients (Kunz et al., 2014). But the mechanism that how immobilization protects the injury site reduces pain and improves healing remains unknown (Huber et al., 2020). Conversely, heterotopic ossification may become more severe in patients with insufficient immobilization and bed rest after fracture injury or joint surgery.

\section{Clinical Risk Factors Physical Factors}

There is a positive correlation between the formation of heterotopic ossification and force application. People who are over-exercised are more likely to develop heterotopic ossification (Jones et al., 2019). The explanation may be that more active people also have a higher probability of injury, excessive stretching of soft tissues leads to abnormal activation and 
differentiation of stem cells in local tissues, or that greater muscle mass leads to mechanical signal stimulation (Coons and Godleski, 2013; Dowdell et al., 2020; Rüdiger et al., 2020). Manifestations of heterotopic ossification due to mechanical stimulation can also occur in the temporomandibular joint (TMJ). Disturbance of occlusal forces will lead to TMJ disorder, while chronic abnormal forces and malposition of the joint will lead to heterotopic ossification of the TMJ (Jensen et al., 2010). Mechanics-based two- and three-dimensional finite element analysis and clinical findings indicate that the occurrence of heterotopic ossification after cervical total intervertebral disc replacement is characterized by a strong correlation with regional stress. Compressive force induces $\mathrm{HO}$ on the uncovered vertebral endplates, while shear force causes $\mathrm{HO}$ in the anterior upper and lower parts of vertebrae (Ganbat et al., 2014; Ganbat et al., 2016).

It is also quite common to apply some physical interventions, such as immobilization, physical therapy, intermittent activity, or massage for convalescent patients. However, the effect of those physical interventions on $\mathrm{HO}$ remains controversial. The transitory periods of forcible passive movements on immobilized arthrosis could produce $\mathrm{HO}$ in the soft tissues around the arthrosis within two to 5 weeks (O'Connor, 1998; Michelsson and Rauschning, 1983). The bone volume of HO was positively correlated with the duration of chronic bed rest and the frequency of forcible movement. Interestingly, $\mathrm{HO}$ was not induced when the limbs were merely immobilized without forcible movement, or merely passively movement without immobilization (Ellerin et al., 1999). Some researchers found that immobilization totally inhibited the formation of $\mathrm{HO}$ (Huber et al., 2020). Some researchers reported that surgery combined with postoperative physical therapy and rehabilitation program was effective to treat patients with heterotopic ossification of the elbow (Salazar et al., 2014). The reasons for this variation may be due to differences in the specific method, time of implementation, and duration of immobilization or rehabilitation exercises, besides the differences in the patients themselves collected in those clinical studies. It takes approximately 5-6 weeks for CTvisible heterotopic ossification to develop at the injury site, and early rehabilitation activities performed at inappropriate time points or approaches that apply additional forces to the injury site will likely result in a higher incidence of $\mathrm{HO}$.

\section{Spinal Cord and Brain Injuries}

Neurogenic HO usually occurs following central nerve injuries, such as spinal cord injuries and cerebral lesions, and the prevalence has been reported to range from 10 to $53 \%$ (Teasell et al., 2010; Ranganathan et al., 2015). However, the mechanism that how the nervous system regulates $\mathrm{HO}$ formation remains incompletely understood. It has been demonstrated that peripheral neurotransmitters influence osteoblast formation, and the cortical bone density can be modulated by mechanistic-neural pathways (Huang et al., 2019; Zhu et al., 2019). Central neural signaling could precisely modulate bone metabolism and homeostasis. Leptin, as well as neuropeptide Y and cannabinoids, play an important role in the neural regulation of bone (Idris et al., 2005; Yue et al., 2016). However, it is unclear whether neural regulation of osteogenesis and osteolysis occurs in the same way as heterotopic ossification. The current researches are primarily devoted to the findings that osteogenic precursor cells in heterotopic ossification originate from the endoneurium and are strongly associated with local neuroinflammation leading to the blood-nerve barrier (BNB) penetration (Lazard et al., 2015; Olmsted-Davis et al., 2017; Davis et al., 2018). In general, thoracic and cervical spine injury can lead to more severe heterotopic ossification, which usually develops caudally at the level of injury, most commonly in the hip joint (Brady et al., 2018). Unlike spinal cord injuries, brain injuries often cause generalized heterotopic ossification, including hip, knee, and elbow or shoulder joints (Garland, 1988).

\section{Empyrosis}

In the case of burn patients, in addition to the typical clinical phenomenon of thermal injury, the occurrence of heterotopic ossification is also frequently observed. Heterotopic ossification is highly probable when the burned area is more than $20 \%$ of the body surface area (Mujtaba et al., 2019). In addition to the burninduced cascade reaction that promotes heterotopic ossification formation, the scar tissue that forms around the periarticular will also limit the range of motion of the joint, which in turn may simultaneously influence heterotopic ossification from a biomechanical approach. Theoretically, the inflammatory cascades due to burns promote heterotopic ossification; the limited fixation due to burning scars may inhibit heterotopic ossification, or the mechanical force from small movements pulls on a large area of tissue due to scars, thus promoting heterotopic ossification. Furthermore, limited joint motion due to scar tissue may also confuse the clinical diagnosis of heterotopic ossification, which could also lead to restricted joint motion. Distinguishing between the two commonly relies on radiographic studies (Suito et al., 2018; Chen et al., 2019).

\section{Surgery}

Surgery that irritates the joint and its surrounding soft tissues may lead to the occurrence of heterotopic ossification. Following hip arthroplasty, the rate of heterotopic ossification occurrence could approach approximately 40\% (Ranganathan et al., 2015). Surgery on the other joints, such as the knee, elbow, and temporomandibular joint, may also result in heterotopic ossification of the soft tissues surrounding them (Meyers et al., 2019). Surgery, especially invasive surgery, can lead to local tissue damage and pathologies such as ischemia and inflammation, which are high-risk factors predisposing to the development of heterotopic ossification. Generally, minimally invasive surgery (MIS), including MIS anterolateral (MIS-AL) and minimally invasive direct anterior approach (AMIS), could reduce the risk of $\mathrm{HO}$ compared with the standard modified anterolateral (STD-Watson-Jones) approach (Hürlimann et al., 2017).

\section{Fracture}

Fractures are an important risk factor for heterotopic ossification. Fractures usually result from trauma, and surgery to treat fractures is in turn invasive trauma to local tissues. $\mathrm{HO}$ following orthopedic injury occurs most frequently after acetabular fractures and elbow fractures. Interestingly, injury 
severity score, sex, and fracture type do not affect this risk, but long-term mechanical ventilation is the specific risk of $\mathrm{HO}$ (Firoozabadi et al., 2014). This is perhaps because of the impact of mechanical ventilation itself on the patient, such as anoxia; or because mechanically ventilated patients are typically bedridden for long periods, which may influence the traditional regulation of bone metabolism and the formation of heterotopic ossification from the mechanism of mechanical signal stimulation.

\section{Management and Treatment Physical Therapy}

The effect of physical therapy on heterotopic ossification is controversial, but physical factors, including postoperative rehabilitation exercises, joint immobilization, and prolonged bed rest, indeed influence heterotopic ossification. It has been shown that complete joint fixation can eliminate heterotopic ossification at the Achilles tendon in the mouse model (Huber et al., 2020). Others, however, believe that early postoperative exercise facilitates recovery and prevents the development of heterotopic ossification (Aronen et al., 2006; Ranganathan et al., 2015; Meyers et al., 2019). Physical therapy and continuous passive motion machines have been used for the postoperative management of total knee arthroplasty, for which a commonly encountered surgical complication is heterotopic ossification. Physical therapy has been found to be moderately beneficial at 3 months after total knee arthroplasty (Lowe et al., 2007; Manrique et al., 2015). A randomized controlled trial also found that physical therapy was superior for total hip replacement management (Mikkelsen et al., 2014). However, burn surgeons often find an increased incidence of $\mathrm{HO}$ in patients who are subjected to overly passive range of motion exercises at the elbow to prevent skin contracture (Meyers et al., 2019). The key to the discrepancy may lie in the duration and timing of the immobilization. In the early post-traumatic phase, immobilization facilitates the normal recovery of local tissues, while repetitive passive movements may lead to an aggravation of local micro-injuries, which in turn may lead to organization and ossification of soft tissues. However, in the late stage of trauma, the local micro-injury and inflammatory environment have been almost recovered, at this time the appropriate passive movement is conducive to the local tissue blood supply and physiological metabolic activities, and is beneficial to the normal recovery of soft tissues. On the contrary, long-term bed rest or immobilization may lead to the deterioration of local microcirculation status, and the abnormal local microenvironment may induce the aberrant differentiation of soft tissue stem cells into bone tissue, resulting in the occurrence of heterotopic ossification.

\section{Pharmaceutical Prophylaxis}

The development of traumatic heterotopic ossification, as previously mentioned, is in part secondary to surgery. It is necessary to take some appropriate clinical interventions to reduce the risk of postoperative heterotopic ossification. Currently, the preventive medications that are more routinely used for HO in clinical practice are NSAIDs and Bisphosphonates
(Ranganathan et al., 2015; Meyers et al., 2019). Essentially, the origin of heterotopic ossification is the abnormal osteogenic differentiation of stem cells in soft tissues. NSAIDs could prevent heterotopic ossification by inhibiting the osteogenic differentiation of progenitor cells (Chang et al., 2007; Chang et al., 2009). However, the negative impact of NSAIDs on fracture healing while preventing heterotopic ossification has to be taken into account. Indomethacin increases the potential risk of longbone nonunion after orthopedic injuries (Marquez-Lara et al., 2016; Duchman et al., 2019). Balancing the risk of heterotopic ossification with malunion fractures is the key to appropriate NSAID delivery.

Bisphosphonates are generally considered to be antiresorptive agents that induce osteoclast apoptosis and inhibit calcification. Yet some studies have indicated that it may have some preventive effect on heterotopic ossification, although this conclusion is still controversial (Vasileiadis et al., 2010; Zaman, 2012). Aside from the first generation, subsequent bisphosphonates generally only affect osteoclasts and thus are less likely to be able to inhibit the production of heterotopic ossification. However, bisphosphonates have indeed been found to be specifically effective in patients with burns and spinal cord injuries (Teasell et al., 2010; Ranganathan et al., 2015). This may be due to the anti-angiogenic effect of bisphosphonates, which reduces the occurrence of $\mathrm{HO}$ by depleting angiogenesis, or because the binding of bisphosphonates to calcium affects the mineralization of the bone matrix.

Some recent studies have also found that non-coding RNAs may have a therapeutic effect on heterotopic ossification, although the effect has yet to be demonstrated in large-scale clinical trials. MicroRNAs targeting DKK1 and vascular endothelial growth factor (VEGF), such as miR-17-5p, can alleviate the heterotopic ossification present in Ankylosing spondylitis (Qin et al., 2019). Similarly, microRNAs that can regulate osteogenic genes, such as miR-203, which targets RUNX2, can also inhibit heterotopic ossification ( $\mathrm{Tu}$ et al., 2016). Further studies of these non-coding RNAs could contribute to the development of medicines that work precisely at the post-transcriptional level for the treatment of heterotopic ossification.

\section{Radiation}

Radiation therapy can be effective in preventing heterotopic ossification after hip arthroplasty. The incidence of heterotopic ossification without radiation after hip arthroplasty is up to $90 \%$, while the rate decreases to about $25 \%$ after radiation therapy (Popovic et al., 2014). Appropriate prophylactic doses generally range from 400 to $800 \mathrm{cGy}$ and are given $24 \mathrm{~h}$ before or $72 \mathrm{~h}$ after surgery, and $700 \mathrm{cGy}(25 \%)$ administered postoperatively was more effective in preventing HO than 400 cGy (42\%) (Popovic et al., 2014; Liu et al., 2017). Higher doses do not demonstrate increased prophylactic benefit, and may bring additional side effects, including progressive soft tissue contracture, delayed wound healing, non-union fracture, joint stiffness, potential oncogenesis, or inhibition of growth of hip implants (Hamid et al., 2010; Milakovic et al., 2015). However, the efficacy of radiation 
TABLE 1 | Cells types contributing to heterotopic ossification.

\begin{tabular}{|c|c|c|}
\hline Study & Cell types & Findings \\
\hline Feng et al. (2020) & Tendon-derived progenitor cells (Ctsk-Cre) & Ctsk could label progenitor cells of $\mathrm{HO}$ in tendon \\
\hline Kan et al. (2018) & Interstitial/perivascular cells (Gli1-Cre) & Gli1-Cre lineage cells contribute to endochondral $\mathrm{HO}$ \\
\hline Agarwal et al. (2017) & Tendon/periosteum/fascia (Scx-Cre) & Scx-cre lineage cells contribute to trauma-induced and BMP-induced $\mathrm{HO}$ \\
\hline Olmsted-Davis et al. (2017) & Endoneurium (Wnt1-CreERT) & $\mathrm{PS}^{+}$and $\mathrm{SP}^{+}$cells from peripheral nerves contribute to $\mathrm{HO}$ \\
\hline Dey et al. (2016) & Endothelial/bone marrow/muscle interstitial cells (Mx1-Cre) & Mx1-Cre lineage cells contribute to intramuscular $\mathrm{HO}$ \\
\hline Agarwal et al. (2015) & Mesenchymal progenitor cells (Nfatc1-Cre) & ca-ACVR $1^{\mathrm{fx} / \mathrm{WT}} / \mathrm{Nfatc1-Cre}{ }^{+}$mice develop heterotopic ossification \\
\hline Regard et al. (2013) & Mesenchymal progenitor cells (Prx1-Cre; Dermo1-Cre; Ap2-Cre) & Loss of Gnas mice resulted in $\mathrm{PHO}$ \\
\hline Kan et al. (2013) & Pericyte/adipocyte/connective tissue interstitium (Glast-CreERT) & Glast-creERT labeled progenitors contribute to $\mathrm{HO}$ at all stages \\
\hline Medici et al. (2010) & Endothelium/muscle satellite cells (Tie2-Cre/VE-Cadherin-Cre) & Endothelium/muscle satellite-derived cells contribute to $\mathrm{HO}$ \\
\hline
\end{tabular}

prevention in joints other than the hip has not been adequately studied.

\section{Surgery}

For heterotopic ossification antecedent to Booker IV Classification, complete surgical resection is achievable as the aberrant bone is free-standing with the hard bone tissue at the joint. Surgical removal is the ultimate treatment for patients who have limited effectiveness with other treatments and are unable to be completely cured (Eęgosz et al., 2019). However, it should be considered that surgical resection itself is an invasive stimulus, which may lead to the recurrence of heterotopic ossification after surgery, especially in susceptible subjects. Otherwise, despite the successful removal of the heterotopic ossified tissue, there is still a risk of recurrence after the surgery.

\section{BIOLOGICAL MECHANISMS OF HO}

The type of ossification that occurs in heterotopic ossification differs depending on the origin of the HO. Among the hereditary HO, Progressive Osseous Heteroplasia (POH) and Albright hereditary osteodystrophy (AHO) are considered to be intramembranous ossification, while fibrodysplasia ossificans progressiva (FOP) is considered to be endochondral ossification (Kaplan and Shore, 2000). This is due to their different pathogenesis. In trauma-induced HO, it is generally accepted that this process occurs through endochondral osteogenesis (Wong et al., 2020). Although the precise mechanism has not been fully investigated, pathological staining such as SOFG on traumatic HO shows that cartilage formation occurs first and then ossification is formed based on it (Yu et al., 2021). However, it is worth exploring whether there is direct differentiation of MSC into osteogenic progenitor cells resulting in intramembranous ossification in traumatic HO. The single-cell sequencing results from the traumatic HO injury site showed that some of the MSCs differentiated into osteoblasts rather than chondrogenic cells (Huber et al., 2020). Moreover, this injury is usually accompanied by nerve and vascular damage. This osteogenesis of neuro- and vascular-derived cells may also affect the frequency of intramembranous vs. endochondral ossification (Wong et al., 2020).

\section{Cell Precursors of HO}

One of the most significant differences between pathological heterotopic ossification and physiological osteogenesis is the distinct cellular source. The cellular origin of physiological osteogenesis is the differentiation of preosteoblast, but the precursor cellular origin of pathological heterotopic ossification has not been fully investigated. Table 1 summarizes the cell types that contribute to heterotopic ossification based on currently published studies. In general, the cellular origin of pathological osteogenesis is not limited to the osteoblast lineage, but potentially results from the pluripotent differentiation of a diverse range of stem cells.

To be more specific, Ctsk was previously found to be able to label osteoclasts and periosteum stem cells. Recently, a subgroup of tendon-derived progenitor cells (TDPCs) was also found to be labeled by Ctsk (Feng et al., 2020). TDPCs, as stem cells in tendon tissue, are capable of multidirectional differentiation and would differentiate towards osteogenesis under certain conditions resulting in heterotopic ossification. In addition, mesenchymal stem cells in tendon areas could also be activated to osteogenic differentiation, which can be labeled by Nfatc1-Cre, Prx1-Cre, and Dermol-Cre. It is possible that some other cells with proliferative capacity may also shift to osteogenic differentiation in some conditions. For example, perivascular cells (Gli1-Cre), $\mathrm{PS}^{+}$and $\mathrm{SP}^{+}$cells from peripheral nerves, and muscle satellite cells (Tie2-Cre/VE-Cadherin-Cre) all contribute to HO. In conclusion, the cellular origin of $\mathrm{HO}$ is relatively complicated, and a variety of cells have the potential to shift to osteogenic differentiation in response to some specific stimulus, which in turn promotes $\mathrm{HO}$ formation.

\section{Inflammation and HO}

Inflammation serves as an important microenvironmental alteration in the development of heterotopic ossification. Trauma leads to a state of local and systemic inflammation, resulting in elevated inflammatory cytokines, such as TNFa, IL$1 \beta$, IL-6, and MCP-1, which could cause abnormal activation of mesenchymal stem cells in the soft tissues (Sung Hsieh et al., 2017). Inflammation-associated cells, such as macrophages and mast cells, also accumulate at the site of trauma-induced heterotopic ossification and promote heterotopic ossification (Convente et al., 2018). Lymphoid tissues also contribute to the cellular niche in Heterotopic Ossification (Loder et al., 2016). The main role of inflammation is to turn MSCs, such 
as normal fibroblast lineage, into the osteogenic lineage, initiating the onset of heterotopic ossification.

\section{Hypoxia and HO}

The hypoxic state of local tissues after trauma may also initiate heterotopic ossification. Regional tissue hypoxia causes the activation of Hypoxia-inducible factors (HIFs), consisting of 1 of 3 a subunits bound to HIF $\beta$ (Meyers et al., 2019). HIFs could increase the production of pro-angiogenic cytokines such as VEGF, facilitating localized pathological bone tissue formation (Dilling et al., 2010; Hwang et al., 2019). The inhibition of HIFs could attenuate $\mathrm{HO}$ formation in experimental models (Agarwal et al., 2016).

\section{Signaling Pathways and HO}

Most of the fundamental research on heterotopic ossification is presently based on traumatic and genetic mouse models. In general, hyperactivation of bone morphogenetic protein (BMP) and consequent cascading activation of activin type- 1 receptor (ACVR1) is thought to lead to abnormal endochondral osteogenesis, resulting in heterotopic ossification. The dysregulation of Hedgehog ( $\mathrm{Hh}$ ) signaling also contributes to many HO. However, recent studies have suggested that this pathological osteogenic process may share similar biological mechanisms with physiological osteogeneses, such as RUNX2, a classical osteogenic transcription factor (Kim et al., 2020). CK2/ HAUSP pathway is a critical regulator of RUNX2 stability because Casein kinase 2 (CK2) phosphorylates RUNX2 and recruits the deubiquitinase herpesvirus-associated ubiquitinspecific protease (HAUSP) to stabilize RUNX2 away from ubiquitin-dependent proteasomal degradation. Meanwhile, regional osteoclast activities are also enhanced during the formation of heterotopic ossification, as the formation of the bone marrow cavities depends on a dynamic balance between osteogenesis and bone resorption. Furthermore, osteogenicosteoclastic crosstalk, such as the transforming growth factorbeta (TGF- $\beta$ ) released after augmented osteoclastic activity that recruits mesenchymal stromal/progenitor cells (MSPCs) in the $\mathrm{HO}$ microenvironment for bone remodeling activities, also plays an important role in heterotopic ossification (Wang et al., 2018). PDGF-BB concentration was also increased during $\mathrm{HO}$ progression. Therefore, the bone formation process of heterotopic ossification is different but correlated to that of normal physiological osteogenesis.

Some proteins that affect bone morphology and bone development also influence the formation of heterotopic ossification. Bone morphogenetic proteins (BMPs) are required for multiple developmental processes, including bone and cartilage formation (Kaliya-Perumal et al., 2020). BMPs bind to ACVR1, which locates on the cell membrane surface phosphorylating SMAD1/5/9(8). Phosphorylated SMAD1/5/ 9(8) combine with SMAD4 and import into the nucleus, regulating transcription that drives endochondral ossification (Nosho et al., 2020). When BMP receptors bonded with Activin A, SMAD2/3 is activated to regulate inflammation (Rautela et al., 2019). The occurrence of FOP is also associated with the $\mathrm{R} 206 \mathrm{H}$ mutant substitution of Acvr1, enhancing the

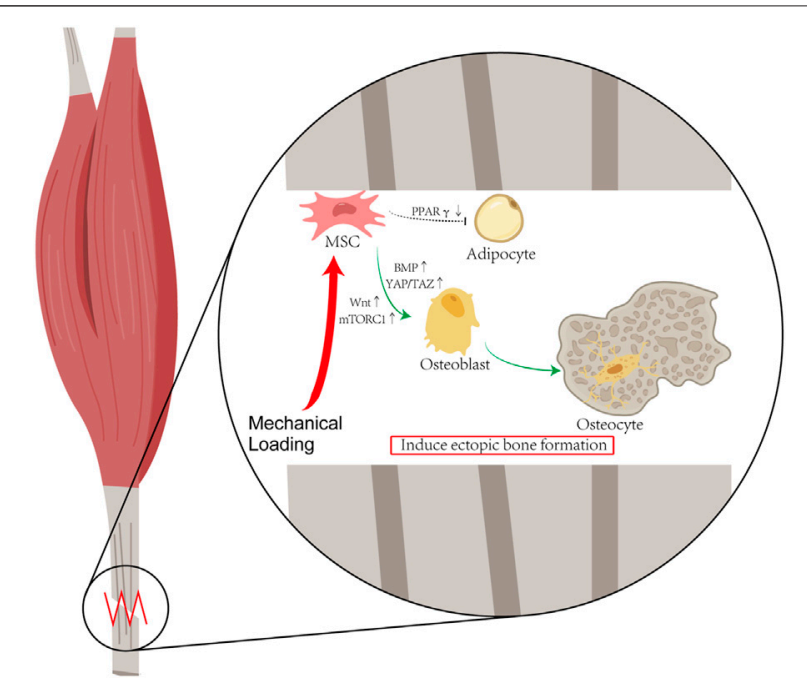

FIGURE 1 | Hypothesis of Mechanical Stimulation of HO: Mechanical stress initiates osteogenic differentiation of mesenchymal stem cells (MSCs) in soft tissue. Stem cell fate of MSCs shifts from favoring lipogenic cells to osteogenic cells under mechanical loading. According to the published literature about $\mathrm{HO}$, after the mechanical loading, the activations of the YAP/ TAZ and mTORC1 pathway enable MSCs to differentiate into osteoblasts, and the decrease in PPAR $\gamma$ expression reduces the differentiation of MSC into adipocytes.

response to various BMP ligands (Alessi Wolken et al., 2018). Retinoic acid receptors (RARs) are morphogens that impact both osteogenesis and chondrogenesis. There is a hypothesis that RAR agonism could impede $\mathrm{HO}$ formation by preventing the differentiation of prechondrogenic cells, and was partly tested in a subcutaneous rBMP2-induced $\mathrm{HO}$ model in mice (Cash et al., 1997; Shimono et al., 2010; Riedl et al., 2020). The Hedgehog ( $\mathrm{Hh}$ ) pathway also plays an important role in $\mathrm{HO}$. Hh protein inhibits the GPCR-like protein Smoothened (SMO) by binding to the Patched (PTCH1) receptor, leading to SMO aggregation in cilia and phosphorylation of the cytoplasmic tail. SMO mediates downstream signaling and induces GLI protein detachment from SUFU. GLI1 and GLI2 proteins translocate to the nucleus to activate the transcription of $\mathrm{Hh}$ target genes (Regard et al., 2013; Feng et al., 2020). From this viewpoint, it can be inferred that biomolecules such as microRNAs, LncRNAs, and exosomes could also regulate heterotopic ossification by influencing some specific key proteins that regulate bone morphology and development, but this remains to further study.

\section{MECHANICS AND HO}

\section{Mechanical Signals of HO}

Heterotopic ossification can be modulated by mechanical signals. It is generally acknowledged that mechanical stress stimulation serves an important function in the physiological osteogenesis process. Osteocytes can sense local mechanical cues and thus induce bone formation, disuse-induced bone loss, and skeletal fragility (Qin et al., 2020). The primary mechanosensors in 
osteocytes include osteocyte cytoskeleton, dendritic processes, integrin-based focal adhesions, connexin-based intercellular junctions, primary cilium, ion channels, and extracellular matrix (Uda et al., 2017). It is now generally accepted that the traditional regulation of bone metabolism is deeply affected by mechanical stimulation signals. Current studies suggest that heterotopic ossification, a pathological osteogenic process, is modulated by mechanical signals as well. Mechanical stress initiates osteogenic differentiation of mesenchymal stem cells (MSCs) in soft tissue. Stem cell fate of MSCs shifts from favoring lipogenic cells to osteogenic cells under mechanical loading (Figure 1).

In the genetic-induced heterotopic ossification murine model, Acvr1 mutant cells change the local microenvironment, resulting in the skewing of the threshold for mechanical stimuli and becoming more sensitive to the fate of chondral/osteogenic lineages (Haupt et al., 2019). Stanley's study revealed that mechanistic signalings of Acvr1 mutant cells in the soft matrix resemble that of non-mutant cells in the hard matrix, and are dependent on RhoA and YAP1 signaling (Stanley et al., 2019). Huber's study found that mechanical stress can be transmitted to mechanosignaling receptors on heterotopic ossified mesenchymal progenitor cells through the extracellular matrix and cell adhesion, such as through focal adhesion kinase signaling and nuclear translocation of the transcription coenzyme TAZ, which regulates the progression of heterotopic ossification (Huber et al., 2020). However, the specific mechanism of Acvr1 in the mechanical signaling process is not clear, and no literature suggests a direct action in the mechanical signaling cascades. Because mutations in Acvr1 result in increased sensitivity to BMP, it is reasonable to believe that the Acvr1 response to mechanical stimulation is BMP-dependent.

Early studies have found that BMP-2, 4, 6, and 7 are differentially expressed depending on the mechanical stimulation (Rui et al., 2011). However, how BMPs can sense mechanical signals has been unclear for a long time, and only recently some studies have made advances. BMP-2 signaling senses mechanical signs because of the cross-talk with YAP/ $\mathrm{TAZ}$ at the transcriptional level. In $\mathrm{C} 2 \mathrm{C} 12$ cells, it was shown that Smad1/5/8 can be phosphorylated and translocated into the nucleus in the presence of BMP-2 signaling alone. However, activation of osteogenic genes requires cytoskeletal tensioninduced nuclear accumulation of YAP/TAZ. BMP-2 signaling responds to mechanical cues by sensing nucleocytoplasmic shuttling of YAP/TAZ (Wei et al., 2020).

YAP and TAZ (also known as WWTR1) are two protooncogene proteins that are widely known as mechanosensors and mechanotransducers in various cell types (Dupont et al., 2011). The link between YAP/TAZ and mechanical signals is extensively explored in physiological osteogenesis as well as in osteogenic lineage. YAP/TAZ translocates from the cytoplasm to the nucleus depending on ECM stiffness in MSCs (Panciera et al., 2017), and mechanical niches trigger $\mathrm{YAP} / \mathrm{TAZ}$ translocation contributing to osteoblastogenesis (Xiong et al., 2018). MST1/2 complexes with the scaffolding protein MOB kinase activator 1 (MOB1) to phosphorylate many proteins involved in chromatin condensation, apoptosis, and proliferation regulation, including cytoplasmic large tumor suppressor kinases 1 and 2 (LATS1 and LATS2). Activated LATS1/2, in turn, binds to YAP/ TAZ and phosphorylates its serine, resulting in its retention in the cytoplasm and non-entry into the nucleus for function (Kovar et al., 2020). This part of YAP/TAZ pathway can interact with multiple signaling pathways at different levels, such as Hippo. In the process of heterotopic ossification, mesenchymal stem cells in soft tissues could be activated for osteogenic differentiation and become osteoblast rather than fibroblast after mechanical stimulation by YAP/TAZ conduction. Moreover, once MSC pluripotent differentiation leads to the initiation of the osteogenic procedure, mechanical stimulation further promotes the proliferation and differentiation of osteoblasts, resulting in enhanced heterotopic ossification ( $\mathrm{Yu}$ et al., 2018). Simultaneously, osteoclast, as well as bone resorption activity, can also be affected by mechanical stresses, and even osteoclastosteoblast crosstalk based on PIEZO1 could occur in response to mechanical forces (Wang et al., 2020). However, it is still unclear whether these osteoclast and osteoblast characteristics of normal bone tissue are completely identical in heterotopic ossification.

LRP5/6 is a key receptor in the Wnt signaling pathway. Wnt signaling plays a central role in the mechanotransduction of bone. But the mechanisms by which wnt signaling senses mechanotransduction signals specifically may be multipathway and multi-level. YAP/TAZ is still an important part of the Wnt pathway to sense mechanical signals. At the cell membrane, YAP/TAZ binds to Axin on LRP6, allowing the recruitment of $\beta$-transducin repeatase containing E3 ubiquitinprotein ligase (BTRC) to the $\beta$-catenin disruption complex (Azzolin et al., 2014). In the cytoplasm, YAP/TAZ binds to the cytoplasmic Wnt signaling transducer disheveled segment polarity protein 1 (DVL1) and inhibits its phosphorylation, thereby abrogating its translocation to the nucleus (Barry et al., 2013). Serine phosphorylated YAP and TAZ can also bind directly to $\beta$-catenin (Zhou et al., 2017). In addition, it can also function as a transcriptional co-activator. How the Wnt pathway specifically senses mechanical signals in bone metabolism has not been completely understood, but there is no doubt that the wnt pathway plays an important role in the biomechanics of bone.

In addition, mTORC1 signaling pathway serves as a mechanosensor modulating HO. Rodgers found that mTORC1 could activate quiescent stem cells into an "alert state" thus responding quickly to injury and stress conditions (Rodgers et al., 2014). The activation of mTORC1 promotes chondrogenesis and osteogenesis. Several studies have demonstrated that mechanical loading could activate the mTORC1 signaling pathway via inducing the phosphorylation of p70 S6 kinase (Lin and Liu, 2019). Chen found mechanical loading modulated $\mathrm{HO}$ of the tendon through the mTORC1 signaling pathway, furthermore, low elongation mechanical loading attenuated $\mathrm{HO}$, while high elongation mechanical loading accelerated $\mathrm{HO}$ in vivo (Chen et al., 2017). Stimulated by mechanistic signaling, mTORC1 activates Sirtuin 1 (Sirt1) in the nucleus. Sirt1 is a histone deacetylase that acts as a novel bone regulator and represses the expression of sclerostin gene SOST, 


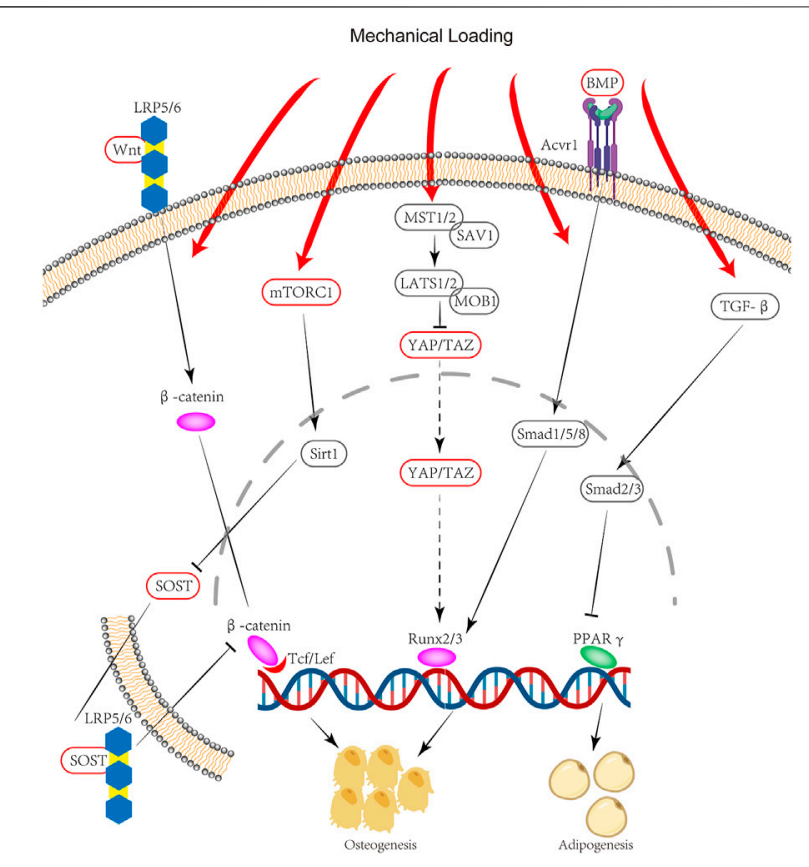

FIGURE 2 | Signaling pathway of $\mathrm{HO}$ due to mechanical stimulation: Mechanical stimulation through $\mathrm{mTORC} 1$ leads to an increase in Sirt1 translocation into the nucleus, followed by a decrease in SOST secretion. SOST can bind to LRP5/6 to inhibit $\beta$-catenin. Mechanical loading can also activate Runx2/3 gene expression through YAP/TAZ. Thus mechanical stimulation promotes osteogenic gene expression through mTORC1 and YAP/TAZ. Meanwhile, mechanical stimulation can inhibit PPAR $\gamma$ gene expression through the TGF- $\beta$ pathway, thereby suppressing lipogenic differentiation. These combined effects lead to a stem cell fate shift.

which is usually regarded as a strong negative regulator of osteoblast differentiation and bone formation (Liu et al., 2019). SOST inhibits $\beta$-catenin and osteogenic gene expression after binding to LRP5/6. Therefore, rapamycin, a selective mTORC1 signaling pathway inhibitor, is a potential therapeutic agent for heterotopic ossification.

\section{Mechanics and Stem Cell Fate}

Mechanical interventions may affect $\mathrm{HO}$ formation by altering stem cell fate. Stem cells are able to sense their mechanical environments through various mechanosensors, including the cytoskeleton, focal adhesions, and primary cilia (Chen and Jacobs, 2013). The cytoskeletal tension could be generated by the interacts between myosin and actin, which is important for mechanically induced osteogenesis of stem cells. Focal adhesion is formed by the adapter proteins linking the cytoskeleton to integrins. Forces are transmitted based on these intact focal adhesions (Nardone et al., 2017). The primary cilium is a single, non-motile, antenna-like transmembrane structure, acting as a microdomain to promote biochemical signaling (Pala et al., 2017). Joint immobilization could reduce mechanotransduction signaling (Kunz et al., 2014). In the immobilized murine model, the fate of mesenchymal progenitor cells was altered. Mobile MPCs expressed more genes related to osteogenesis and chondrogenesis, such as
Sox9, Runx2, Spp1, and differentiated more into osteogenic cells; immobile MPCs expressed more genes related to lipogenesis, e.g. Fabp4, Pltp, Lrp1, and differentiated more into lipogenic cells (Huber et al., 2020). In the osteogenic-lipogenic fate shifting of MSCs caused by mechanical stimulation, sclerostin signaling potentially serves as a significant regulator. Unloading makes the expression of the sclerostin increase, which downregulates two key osteogenic procedures: Wnt/ $\beta$-catenin signaling and YAP/TAZ transcriptional activity. The crosstalks between $\mathrm{Wnt} / \beta$-catenin and PPAR $\gamma$ influence the physiological balance between osteogenesis and adipogenesis (Benayahu et al., 2019). As the MSCs are mechanically stimulated and favor osteogenic differentiation, heterotopic ossification becomes severe. Conversely, when they favor lipogenic differentiation, the amount of heterotopic ossified bone decreases. Therefore, joint immobility after injury promotes adipogenesis rather than osteogenesis, leading to reduced $\mathrm{HO}$ formation. And the use of pharmacologic inhibitors altering mechanical signaling may prove to be an effective therapy that spontaneously induces adipogenesis at sites prone to osteogenesis. The accumulation of fatty tissue in the joint near the site of injury is much less severe than $\mathrm{HO}$, leading to a more favorable outcome (McTighe and Chernev, 2014).

Mechanical loading has also been demonstrated to cause stem cell fate shift at the cellular level (Figure 2). Mechanical loading appears to favor osteogenesis whereas unloading conditions seem to promote adipogenesis. Passive stimuli including stiffness and viscoelasticity, as well as active stimuli including tensile/ compressive stress and fluid shear stress, can affect cells through the extracellular matrix (Benayahu et al., 2019). Mechanical signals are conducted from the extracellular matrix through the cytoskeleton to regulate intracellular actions. Some important signaling pathways interact with mechanistic signals. For example, Wnt ligand binding to low-density lipoprotein receptor-related protein 5/6 (LRP5/6) coreceptors results in the translocation of $\beta$-catenin to the nucleus and the enhanced transcription of genes that govern osteogenesis, and its interaction with the Hippo pathway that governs the activity of YAP/TAZ, which is regarded as an important mechanistic signaling transcription factor (Benayahu et al., 2019; García de Herreros and Duñach, 2019). Even cells that already have terminally differentiated into the myogenic lineage may be reconverted to the osteoblast lineage under certain conditions: $\mathrm{C} 2 \mathrm{C} 12$, a myoblast cell line, can be converted to osteoblasts under the combined effect of BMP and mechanical stimulation (Wei et al., 2020). Although these studies demonstrate that cells of other lineages are capable of osteogenic differentiation, it is not clear whether the same phenotype occurs in vivo, resulting in heterotopic ossification.

Beyond biological experiments, a significant influence of local loading on the formation of heterotopic ossification has been found through the mechanobiological algorithm system. By designing a computational model of physiology that takes into account both mechanical and biological factors, Rosenberg found that modifications to the mechanical environment significantly alter the shape and production of heterotopic bone. Adjustment of load orientation, skin material characteristics, and location of 
maximum trauma resulted in four characteristic $\mathrm{HO}$ types. Simulation of negative pressure dressings and tourniquet application also served to highlight the behavioral characteristics of HO (Rosenberg and Bull, 2018). Still, the mechanobiological algorithm system needs further development to make it more compatible with the real world.

These basic studies suggest that mechanical signals contribute to the formation and development of heterotopic ossification, not only initiate heterotopic ossification through the activation of pluripotent differentiation of MSCs, but also influence the osteogenic program during $\mathrm{HO}$ by affecting osteocytes, osteoblasts, and osteoclasts. However, there are only a few studies related to mechanical stimulation and heterotopic ossification. Representative basic studies have only applied fixed models for attenuated mechanical stimulation, but elaborate force-added models also need to be investigated. Relevant clinical studies are even more lacking. Further studies in this direction would have guiding values for the development of new drug targets for the treatment of $\mathrm{HO}$, as well as for the development of more effective clinical methods of physical therapy and prophylaxis for HO.

In summary, the effects of mechanics on heterotopic ossification could be considered from early, middle, and late stages, respectively. In the early stage of $\mathrm{HO}$, mechanical stimulation may activate pluripotent differentiation of MSCs in soft tissues, e.g., mTORC1 could activate quiescent stem cells into an "alert state", and promote chondrogenesis and osteogenesis, leading to $\mathrm{HO}$ initiation. Mechanical stimulation can alter stem cell fate, causing chromatin regions around osteogenic genes to open. This results in more expression of osteogenic-related proteins and promotes stem cell differentiation toward osteogenesis. Clinically, early posttrauma immobilization can attenuate or even prevent heterotopic ossification. In the middle stage of $\mathrm{HO}$, which means heterotopic ossification has been triggered and pathologic ossification is in the process of formation. Since physiological osteogenesis and pathological osteogenesis have some commonalities, they both require stem cells to differentiate into osteoblasts, and the eventual ossifications are dependent on the function of osteoblasts performing osteogenic functions. Many fundamental signaling pathway, such as CK2/ HAUSP/RUNX2 are necessary for both physiologic bone formation and $\mathrm{HO}$. It can be assumed that the effects of mechanics on HO may be similar to that on the osteogenesis process. From the clinical perspective, patients at this stage may still need as much bed rest as possible to avoid stress on the trauma site and to prevent pathological osteogenesis. Conversely, for the late stage, prolonged immobilization may instead lead to

\section{REFERENCES}

Ackerman, L. V. (1958). Extra-Osseous Localized Non-neoplastic Bone and Cartilage Formation (So-Called Myositis Ossificans). J. Bone Jt. Surg. 40, 279-298. doi:10.2106/00004623-195840020-00004

Agarwal, S., Loder, S. J., Brownley, C., Eboda, O., Peterson, J. R., Hayano, S., et al. (2015). BMP Signaling Mediated by Constitutively Active Activin Type 1 local tissue inflammation and hypoxia, both of which are risk factors for heterotopic ossification, and may lead to tissue ischemia and necrosis along with malfunctioning. Therefore, for patients potentially suffering from heterotopic ossification in the initial stages of injury, early and adequate immobilization is essential to avoid stress on the injured area. For those patients who have been adequately immobilized after trauma, appropriate rehabilitation exercises are recommended in the late stages to prevent heterotopic ossification as well as promote functional recovery.

\section{SUMMARY}

$\mathrm{HO}$ is a diverse pathologic process. We still do not fully understand the cellular origin, pathogenesis, and underlying mechanisms of $\mathrm{HO}$, and have not yet developed a specific treatment for $\mathrm{HO}$ beyond surgical resection. $\mathrm{HO}$ as a pathological osteogenic activity involving pluripotent differentiation of stem cells has many remaining aspects to be explored, although it has similarities to physiological osteogenic activity in some ways. This paper reviews the features of heterotopic ossification according to the established literature, with particular emphasis on the effect of mechanical stimuli on HO. However, the specific biological mechanism of this effect needs to be further investigated.

\section{AUTHOR CONTRIBUTIONS}

CL contributed to concepts, review article revising, and final approval of article; YX draft the review article and prepared the figures; $\mathrm{KC}$ and $\mathrm{CH}$ helped in preparing the figures. $\mathrm{MeH}, \mathrm{WH}$, $\mathrm{YJ}, \mathrm{JH}, \mathrm{MiH}, \mathrm{NZ}, \mathrm{LL}$, and RL and helped in the writing and submission of the article. All authors have read and agreed to the published version of the manuscript.

\section{FUNDING}

This work was supported by the National Key R\&D Program of China (Grant No. 2019YFA0111900), the National Natural Science Foundation of China (Grant Nos 81922017, 81873669, 81802209), the Hunan Province Natural Science Foundation of China (Grant No. 2018JJ3863), Hunan Provincial Science and Technology Department (Grant No. 2018RS3030), the Innovation Driven Project of Central South University (Grant No. 20170033010017).

Receptor (ACVR1) Results in Ectopic Bone Formation Localized to Distal Extremity Joints. Develop. Biol. 400, 202-209. doi:10.1016/j.ydbio.2015.02.011 Agarwal, S., Loder, S., Brownley, C., Cholok, D., Mangiavini, L., Li, J., et al. (2016). Inhibition of Hifla Prevents Both Trauma-Induced and Genetic Heterotopic Ossification. Proc. Natl. Acad. Sci. USA 113, E338-E347. doi:10.1073/pnas.1515397113

Agarwal, S., Loder, S. J., Cholok, D., Peterson, J., Li, J., Breuler, C., et al. (2017). Scleraxis-Lineage Cells Contribute to Ectopic Bone Formation in Muscle and Tendon. Stem Cells 35, 705-710. doi:10.1002/stem.2515 
Alessi Wolken, D. M., Idone, V., Hatsell, S. J., Yu, P. B., and Economides, A. N. (2018). The Obligatory Role of Activin A in the Formation of Heterotopic Bone in Fibrodysplasia Ossificans Progressiva. Bone 109, 210-217. doi:10.1016/ j.bone.2017.06.011

Aronen, J. G., Garrick, J. G., Chronister, R. D., and McDevitt, E. R. (2006). Quadriceps Contusions: Clinical Results of Immediate Immobilization in 120 Degrees of Knee Flexion. Clin. J. Sport Med. 16, 383-387. doi:10.1097/ 01.jsm.0000244605.34283.94

Azzolin, L., Panciera, T., Soligo, S., Enzo, E., Bicciato, S., Dupont, S., et al. (2014). YAP/TAZ Incorporation in the $\beta$-Catenin Destruction Complex Orchestrates the Wnt Response. Cell 158, 157-170. doi:10.1016/j.cell.2014.06.013

Balboni, T. A., Gobezie, R., and Mamon, H. J. (2006). Heterotopic Ossification: Pathophysiology, Clinical Features, and the Role of Radiotherapy for Prophylaxis. Int. J. Radiat. Oncol. Biol. Phys. 65, 1289-1299. doi:10.1016/ j.ijrobp.2006.03.053

Barry, E. R., Morikawa, T., Butler, B. L., Shrestha, K., de la Rosa, R., Yan, K. S., et al. (2013). Restriction of Intestinal Stem Cell Expansion and the Regenerative Response by YAP. Nature 493, 106-110. doi:10.1038/nature11693

Baujat, G., Choquet, R., Bouée, S., Jeanbat, V., Courouve, L., Ruel, A., et al. (2017). Prevalence of Fibrodysplasia Ossificans Progressiva (FOP) in France: an Estimate Based on a Record Linkage of Two National Databases. Orphanet J. Rare Dis. 12, 123. doi:10.1186/s13023-017-0674-5

Benayahu, D., Wiesenfeld, Y., and Sapir-Koren, R. (2019). How Is Mechanobiology Involved in Mesenchymal Stem Cell Differentiation toward the Osteoblastic or Adipogenic Fate? J. Cel Physiol. 234, 12133-12141. doi:10.1002/jcp.28099

Botman, E., Treurniet, S., Lubbers, W. D., Schwarte, L. A., Schober, P. R., Sabelis, L., et al. (2020). When Limb Surgery Has Become the Only Life-Saving Therapy in FOP: A Case Report and Systematic Review of the Literature. Front. Endocrinol. 11, 570. doi: $10.3389 /$ fendo.2020.00570

Brady, R. D., Shultz, S. R., McDonald, S. J., and O’Brien, T. J. (2018). Neurological Heterotopic Ossification: Current Understanding and Future Directions. Bone 109, 35-42. doi:10.1016/j.bone.2017.05.015

Brooker, A. F., Bowerman, J. W., Robinson, R. A., and Riley, L. H., Jr (1973). Ectopic Ossification Following Total Hip Replacement. J. Bone Jt. Surg. 55, 1629-1632. doi:10.2106/00004623-197355080-00006

Cash, D. E., Bock, C. B., Schughart, K., Linney, E., and Underhill, T. M. (1997). Retinoic Acid Receptor a Function in Vertebrate Limb Skeletogenesis: a Modulator of Chondrogenesis. J. Cel Biol. 136, 445-457. doi:10.1083/ jcb.136.2.445

Chang, J.-K., Li, C.-J., Wu, S.-C., Yeh, C.-H., Chen, C.-H., Fu, Y.-C., et al. (2007). Effects of Anti-inflammatory Drugs on Proliferation, Cytotoxicity and Osteogenesis in Bone Marrow Mesenchymal Stem Cells. Biochem. Pharmacol. 74, 1371-1382. doi:10.1016/j.bcp.2007.06.047

Chang, J.-K., Li, C.-J., Liao, H.-J., Wang, C.-K., Wang, G.-J., and Ho, M.-L. (2009). Anti-inflammatory Drugs Suppress Proliferation and Induce Apoptosis through Altering Expressions of Cell Cycle Regulators and Pro-apoptotic Factors in Cultured Human Osteoblasts. Toxicology 258, 148-156. doi:10.1016/j.tox.2009.01.016

Chen, J. C., and Jacobs, C. R. (2013). Mechanically Induced Osteogenic Lineage Commitment of Stem Cells. Stem Cel Res. Ther. 4, 107. doi:10.1186/scrt318

Chen, G., Jiang, H., Tian, X., Tang, J., Bai, X., Zhang, Z., et al. (2017). Mechanical Loading Modulates Heterotopic Ossification in Calcific Tendinopathy through the mTORC1 Signaling Pathway. Mol. Med. Rep. 16, 5901-5907. doi:10.3892/ mmr.2017.7380

Chen, J.-Y., Fu, C.-W., Ho, H.-Y., and Lu, Y.-C. (2019). Surgical Treatment of Postburn Heterotopic Ossification Around the Elbow. Medicine (Baltimore) 98, e14403. doi:10.1097/md.0000000000014403

Cheng, L., Long, H.-T., Sun, B.-H., Zhao, S.-s., and Zhu, Y. (2017). The Efficacy of a Multimodal Analgesia Protocol in Preventing Heterotopic Ossification after Acetabular Fractures Surgery. Int. J. Clin. Pharm. 39, 826-830. doi:10.1007/ s11096-017-0476-9

Cipriano, C. A., Pill, S. G., and Keenan, M. A. (2009). Heterotopic Ossification Following Traumatic Brain Injury and Spinal Cord Injury. J. Am. Acad. Orthop. Surg. 17, 689-697. doi:10.5435/00124635-200911000-00003

Convente, M. R., Chakkalakal, S. A., Yang, E., Caron, R. J., Zhang, D., Kambayashi, T., et al. (2018). Depletion of Mast Cells and Macrophages Impairs Heterotopic Ossification in an Acvr1R206H Mouse Model of Fibrodysplasia Ossificans Progressiva. J. Bone Miner Res. 33, 269-282. doi:10.1002/jbmr.3304
Coons, D., and Godleski, M. (2013). Range of Motion Exercises in the Setting of Burn-Associated Heterotopic Ossification at the Elbow: Case Series and Discussion. Burns 39, e34-e38. doi:10.1016/j.burns.2012.10.014

Crowgey, E. L., Wyffels, J. T., Osborn, P. M., Wood, T. T., and Edsberg, L. E. (2018). A Systems Biology Approach for Studying Heterotopic Ossification: Proteomic Analysis of Clinical Serum and Tissue Samples. Genomics Proteomics Bioinformatics 16, 212-220. doi:10.1016/j.gpb.2018.04.006

Davis, E. L., Davis, A. R., Gugala, Z., and Olmsted-Davis, E. A. (2018). Is Heterotopic Ossification Getting Nervous?: The Role of the Peripheral Nervous System in Heterotopic Ossification. Bone 109, 22-27. doi:10.1016/ j.bone.2017.07.016

Dey, D., Bagarova, J., Hatsell, S. J., Armstrong, K. A., Huang, L., Ermann, J., et al. (2016). Two Tissue-Resident Progenitor Lineages Drive Distinct Phenotypes of Heterotopic Ossification. Sci. Transl. Med. 8, 366ra163. doi:10.1126/ scitranslmed.aaf 1090

Dilling, C. F., Wada, A. M., Lazard, Z. W., Salisbury, E. A., Gannon, F. H., Vadakkan, T. J., et al. (2010). Vessel Formation Is Induced Prior to the Appearance of Cartilage in BMP-2-Mediated Heterotopic Ossification. J. Bone Miner. Res. 25, 1147-1156. doi:10.1359/jbmr.091031

Dowdell, J. E., Kim, J. S., Mikhail, C., Overley, S. C., Levin, J. M., McAnany, S. J., et al. (2020). The Rate of Heterotopic Ossification Following Cervical Disc Arthroplasty. Spine 45, E1197-E1202. doi:10.1097/brs.0000000000003524

Duchman, K. R., Lemmex, D. B., Patel, S. H., Ledbetter, L., Garrigues, G. E., and Riboh, J. C. (2019). The Effect of Non-steroidal Anti-inflammatory Drugs on Tendon-To-Bone Healing: A Systematic Review with Subgroup Meta-Analysis. Iowa Orthop. J. 39, 107-119.

Dupont, S., Morsut, L., Aragona, M., Enzo, E., Giulitti, S., Cordenonsi, M., et al. (2011). Role of YAP/TAZ in Mechanotransduction. Nature 474, 179-183. doi:10.1038/nature10137

Ellerin, B. E., Helfet, D., Parikh, S., Hotchkiss, R. N., Levin, N., Nisce, L., et al. (1999). Current Therapy in the Management of Heterotopic Ossification of the Elbow. Am. J. Phys. Med. Rehabil. 78, 259-271. doi:10.1097/00002060199905000-00016

Elmas, F., and Shrestha, B. L. (2017). Eagle's Syndrome. N. Engl. J. Med. 377, e18. doi:10.1056/nejmicm 1703542

Feng, H., Xing, W., Han, Y., Sun, J., Kong, M., Gao, B., et al. (2020). Tendonderived Cathepsin K-Expressing Progenitor Cells Activate Hedgehog Signaling to Drive Heterotopic Ossification. J. Clin. Invest. 130, 6354. doi:10.1172/ jci132518

Firoozabadi, R., O'Mara, T. J., Swenson, A., Agel, J., Beck, J. D., and Routt, M. (2014). Risk Factors for the Development of Heterotopic Ossification after Acetabular Fracture Fixation. Clin. Orthop. Relat. Res. 472, 3383-3388. doi:10.1007/s11999-014-3719-2

Forsberg, J. A., Pepek, J. M., Wagner, S., Wilson, K., Flint, J., Andersen, R. C., et al. (2009). Heterotopic Ossification in High-Energy Wartime Extremity Injuries: Prevalence and Risk Factors. J. Bone Jt. Surg. Am. 91, 1084-1091. doi:10.2106/ jbjs.h.00792

Forsberg, J. A., Davis, T. A., Elster, E. A., and Gimble, J. M. (2014). Burned to the Bone. Sci. Transl. Med. 6, 255fs37. doi:10.1126/scitranslmed.3010168

Ganbat, D., Kim, K., Jin, Y. J., and Kim, Y. H. (2014). Heterotopic Ossification in Cervical Total Disk Replacement: a Finite Element Analysis. Proc. Inst. Mech. Eng. H 228, 200-205. doi:10.1177/0954411914522024

Ganbat, D., Kim, Y. H., Kim, K., Jin, Y. J., and Park, W. M. (2016). Effect of Mechanical Loading on Heterotopic Ossification in Cervical Total Disc Replacement: a Three-Dimensional Finite Element Analysis. Biomech. Model. Mechanobiol. 15, 1191-1199. doi:10.1007/s10237-015-0752-3

García de Herreros, A., and Duñach, M. (2019). Intracellular Signals Activated by Canonical Wnt Ligands Independent of GSK3 Inhibition and $\beta$-Catenin Stabilization. Cells 8, 1148. doi:10.3390/cells8101148

Garland, D. E. (1988). Clinical Observations on Fractures and Heterotopic Ossification in the Spinal Cord and Traumatic Brain Injured Populations. Clin. Orthop. Relat. Res. 233, 86-101. doi:10.1097/00003086-198808000-00011

Gomez-Puerto, M. C., Iyengar, P. V., García de Vinuesa, A., Ten Dijke, P., and Sanchez-Duffhues, G. (2019). Bone Morphogenetic Protein Receptor Signal Transduction in Human Disease. J. Pathol. 247, 9-20. doi:10.1002/path.5170

Hamid, N., Ashraf, N., Bosse, M. J., Connor, P. M., Kellam, J. F., Sims, S. H., et al. (2010). Radiation Therapy for Heterotopic Ossification Prophylaxis Acutely after Elbow Trauma. J. Bone Jt. Surg Am 92, 2032-2038. doi:10.2106/jbjs.i.01435 
Hastings, H., 2nd, and Graham, T. J. (1994). The Classification and Treatment of Heterotopic Ossification about the Elbow and Forearm. Hand Clin. 10, 417-437. doi:10.1016/s0749-0712(21)01180-x

Haupt, J., Stanley, A., McLeod, C. M., Cosgrove, B. D., Culbert, A. L., Wang, L., et al. (2019). ACVR1R206H FOP Mutation Alters Mechanosensing and Tissue Stiffness during Heterotopic Ossification. MBoC 30, 17-29. doi:10.1091/ mbc.e18-05-0311

Huang, S., Li, Z., Liu, Y., Gao, D., Zhang, X., Hao, J., et al. (2019). Neural Regulation of Bone Remodeling: Identifying Novel Neural Molecules and Pathways between Brain and Bone. J. Cel Physiol. 234, 5466-5477. doi:10.1002/jcp.26502

Huber, A. K., Patel, N., Pagani, C. A., Marini, S., Padmanabhan, K. R., Matera, D. L., et al. (2020). Immobilization after Injury Alters Extracellular Matrix and Stem Cell Fate. J. Clin. Invest. 130, 5444-5460. doi:10.1172/jci136142

Hürlimann, M., Schiapparelli, F. F., Rotigliano, N., Testa, E., Amsler, F., and Hirschmann, M. T. (2017). Influence of Surgical Approach on Heterotopic Ossification after Total Hip Arthroplasty - Is Minimal Invasive Better? A Case Control Study. BMC Musculoskelet. Disord. 18, 27. doi:10.1186/s12891-0171391-x

Hwang, C., Marini, S., Huber, A. K., Stepien, D. M., Sorkin, M., Loder, S., et al. (2019). Mesenchymal VEGFA Induces Aberrant Differentiation in Heterotopic Ossification. Bone Res. 7, 36. doi:10.1038/s41413-019-0075-6

Idris, A. I., van 't Hof, R. J., Greig, I. R., Ridge, S. A., Baker, D., Ross, R. A., et al. (2005). Regulation of Bone Mass, Bone Loss and Osteoclast Activity by Cannabinoid Receptors. Nat. Med. 11, 774-779. doi:10.1038/nm1255

Jensen, A. W., Viozzi, C. F., and Foote, R. L. (2010). Long-term Results of Radiation Prophylaxis for Heterotopic Ossification in the Temporomandibular Joint. J. Oral Maxillofac. Surg. 68, 1100-1105. doi:10.1016/j.joms.2009.12.018

Jones, N. A., Bentley, B. C., and Wahl, L. (2019). Nonsurgical Management of Heterotopic Ossification in a Runner. J. Orthop. Sports Phys. Ther. 49, 676. doi:10.2519/jospt.2019.8491

Kaliya-Perumal, A. K., Carney, T. J., and Ingham, P. W. (2020). Fibrodysplasia Ossificans Progressiva: Current Concepts from Bench to Bedside. Dis. Model. Mech. 13, dmm046441. doi:10.1242/dmm.046441

Kan, L., Peng, C.-Y., McGuire, T. L., and Kessler, J. A. (2013). Glast-expressing Progenitor Cells Contribute to Heterotopic Ossification. Bone 53, 194-203. doi:10.1016/j.bone.2012.12.008

Kan, C., Chen, L., Hu, Y., Ding, N., Li, Y., McGuire, T. L., et al. (2018). Gli1-labeled Adult Mesenchymal Stem/progenitor Cells and Hedgehog Signaling Contribute to Endochondral Heterotopic Ossification. Bone 109, 71-79. doi:10.1016/ j.bone.2017.06.014

Kaplan, F. S., and Shore, E. M. (2000). Progressive Osseous Heteroplasia. J. Bone Miner Res. 15, 2084-2094. doi:10.1359/jbmr.2000.15.11.2084

Kaplan, F. S., Hahn, G. V., and Zasloff, M. A. (1994). Heterotopic Ossification: Two Rare Forms and what They Can Teach Us. J. Am. Acad. Orthop. Surg. 2, 288-296. doi:10.5435/00124635-199409000-00007

Kim, J.-M., Yang, Y.-S., Park, K. H., Ge, X., Xu, R., Li, N., et al. (2020). A RUNX2 Stabilization Pathway Mediates Physiologic and Pathologic Bone Formation. Nat. Commun. 11, 2289. doi:10.1038/s41467-02016038-6

Ko, J.-W. K., Tompson, J. D., Sholder, D. S., Black, E. M., and Abboud, J. A. (2016). Heterotopic Ossification of the Long Head of the Triceps after Reverse Total Shoulder Arthroplasty. J. Shoulder Elbow Surg. 25, 1810-1815. doi:10.1016/ j.jse.2016.03.006

Kovar, H., Bierbaumer, L., and Radic-Sarikas, B. (2020). The YAP/TAZ Pathway in Osteogenesis and Bone Sarcoma Pathogenesis. Cells 9, 972. doi:10.3390/ cells 9040972

Kunz, R. I., Coradini, J. G., Silva, L. I., Bertolini, G. R. F., Brancalhão, R. M. C., and Ribeiro, L. F. C. (2014). Effects of Immobilization and Remobilization on the Ankle Joint in Wistar Rats. Braz. J. Med. Biol. Res. 47, 842-849. doi:10.1590/ 1414-431x20143795

Lazard, Z. W., Olmsted-Davis, E. A., Salisbury, E. A., Gugala, Z., Sonnet, C., Davis, E. L., et al. (2015). Osteoblasts Have a Neural Origin in Heterotopic Ossification. Clin. Orthop. Relat. Res. 473, 2790-2806. doi:10.1007/s11999015-4323-9

Łęgosz, P., Otworowski, M., Sibilska, A., Starszak, K., Kotrych, D., Kwapisz, A., et al. (2019). Heterotopic Ossification: A Challenging Complication of Total Hip Arthroplasty: Risk Factors, Diagnosis, Prophylaxis, and Treatment. Biomed. Res. Int. 2019, 3860142. doi:10.1155/2019/3860142
Lin, S.-S., and Liu, Y.-W. (2019). Mechanical Stretch Induces mTOR Recruitment and Activation at the Phosphatidic Acid-Enriched Macropinosome in Muscle Cell. Front. Cel Dev. Biol. 7, 78. doi:10.3389/fcell.2019.00078

Liu, J. Z., Frisch, N. B., Barden, R. M., Rosenberg, A. G., Silverton, C. D., and Galante, J. O. (2017). Heterotopic Ossification Prophylaxis after Total Hip Arthroplasty: Randomized Trial of 400 vs 700 cGy. J. Arthroplasty 32, 1328-1334. doi:10.1016/j.arth.2016.10.030

Liu, W., Wang, Z., Yang, J., Wang, Y., Li, K., Huang, B., et al. (2019). Osteocyte TSC1 Promotes Sclerostin Secretion to Restrain Osteogenesis in Mice. Open Biol. 9, 180262. doi:10.1098/rsob.180262

Loder, S., Agarwal, S., Sorkin, M., Breuler, C., Li, J., Peterson, J., et al. (2016). Lymphatic Contribution to the Cellular Niche in Heterotopic Ossification. Ann. Surg. 264, 1174-1180. doi:10.1097/sla.0000000000001619

Lowe, C. J. M., Barker, K. L., Dewey, M., and Sackley, C. M. (2007). Effectiveness of Physiotherapy Exercise after Knee Arthroplasty for Osteoarthritis: Systematic Review and Meta-Analysis of Randomised Controlled Trials. BMJ 335, 812. doi:10.1136/bmj.39311.460093.be

Maender, C., Sahajpal, D., and Wright, T. W. (2010). Treatment of Heterotopic Ossification of the Elbow Following Burn Injury: Recommendations for Surgical Excision and Perioperative Prophylaxis Using Radiation Therapy. J. Shoulder Elbow Surg. 19, 1269-1275. doi:10.1016/j.jse.2010.05.029

Malca, N., Serror, K., Mimoun, M., Chatelain, S., Kaplan, J., Chaouat, M., et al. (2018). Our 35 years' Experience on Postburn Heterotopic Ossification: A Three-step Treatment. Ann. de Chirurgie Plastique Esthétique 63, 316-322. doi:10.1016/j.anplas.2017.11.009

Manrique, J., Gomez, M. M., and Parvizi, J. (2015). Stiffness after Total Knee Arthroplasty. J. Knee Surg. 28, 119-126. doi:10.1055/s-0034-1396079

Marquez-Lara, A., Hutchinson, I. D., Nuñez, F., Jr., Smith, T. L., and Miller, A. N. (2016). Nonsteroidal Anti-inflammatory Drugs and Bone-Healing: A Systematic Review of Research Quality. JBJS Rev. 4, e4. doi:10.2106/ JBJS.RVW.O.00055

McTighe, S., and Chernev, I. (2014). Intramuscular Lipoma: a Review of the Literature. Orthop. Rev. (Pavia) 6, 5618. doi:10.4081/or.2014.5618

Medici, D., Shore, E. M., Lounev, V. Y., Kaplan, F. S., Kalluri, R., and Olsen, B. R. (2010). Conversion of Vascular Endothelial Cells into Multipotent Stem-like Cells. Nat. Med. 16, 1400-1406. doi:10.1038/nm.2252

Medina, A., Shankowsky, H., Savaryn, B., Shukalak, B., and Tredget, E. E. (2014). Characterization of Heterotopic Ossification in Burn Patients. J. Burn Care Res. 35, 251-256. doi:10.1097/bcr.0b013e3182957768

Medina, A., Ma, Z., Varkey, M., Liu, H., Iwashina, T., Ding, J., et al. (2015). Fibrocytes Participate in the Development of Heterotopic Ossification. J. Burn Care Res. 36, 394-404. doi:10.1097/bcr.0000000000000102

Meyers, C., Lisiecki, J., Miller, S., Levin, A., Fayad, L., Ding, C., et al. (2019). Heterotopic Ossification: A Comprehensive Review. JBMR Plus 3, e10172. doi:10.1002/jbm4.10172

Michelsson, J.-E., and Rauschning, W. (1983). Pathogenesis of Experimental Heterotopic Bone Formation Following Temporary Forcible Exercising of Immobilized Limbs. Clin. Orthop. Relat. Res. 176, 265-272. doi:10.1097/ 00003086-198306000-00039

Mikkelsen, L. R., Mechlenburg, I., Søballe, K., Jørgensen, L. B., Mikkelsen, S., Bandholm, T., et al. (2014). Effect of Early Supervised Progressive Resistance Training Compared to Unsupervised home-based Exercise after Fast-Track Total Hip Replacement Applied to Patients with Preoperative Functional Limitations. A Single-Blinded Randomised Controlled Trial. Osteoarthr. Cartil. 22, 2051-2058. doi:10.1016/j.joca.2014.09.025

Milakovic, M., Popovic, M., Raman, S., Tsao, M., Lam, H., and Chow, E. (2015). Radiotherapy for the Prophylaxis of Heterotopic Ossification: A Systematic Review and Meta-Analysis of Randomized Controlled Trials. Radiother. Oncol. 116, 4-9. doi:10.1016/j.radonc.2015.05.022

Mujtaba, B., Taher, A., Fiala, M. J., Nassar, S., Madewell, J. E., Hanafy, A. K., et al. (2019). Heterotopic Ossification: Radiological and Pathological Review. Radiol. Oncol. 53, 275-284. doi:10.2478/raon-2019-0039

Naraghi, F. F., DeCoster, T. A., Moneim, M. S., Miller, R. A., and Rivero, D. (1996). Heterotopic Ossification. Orthopedics 19, 145-152. doi:10.3928/0147-744719960201-10

Nardone, G., Oliver-De La Cruz, J., Vrbsky, J., Martini, C., Pribyl, J., Skládal, P., et al. (2017). YAP Regulates Cell Mechanics by Controlling Focal Adhesion Assembly. Nat. Commun. 8, 15321. doi:10.1038/ncomms15321 
Nosho, S., Tosa, I., Ono, M., Hara, E. S., Ishibashi, K., Mikai, A., et al. (2020). Distinct Osteogenic Potentials of BMP-2 and FGF-2 in Extramedullary and Medullary Microenvironments. Int. J. Mol. Sci. 21, 7967. doi:10.3390/ ijms 21217967

O'Connor, J. P. (1998). Animal Models of Heterotopic Ossification. Clin. Orthop. Relat. Res., 71-80.

Olmsted-Davis, E. A., Salisbury, E. A., Hoang, D., Davis, E. L., Lazard, Z., Sonnet, C., et al. (2017). Progenitors in Peripheral Nerves Launch Heterotopic Ossification. Stem Cell Transl. Med. 6, 1109-1119. doi:10.1002/sctm.16-0347

Pala, R., Alomari, N., and Nauli, S. M. (2017). Primary Cilium-dependent Signaling Mechanisms. Int. J. Mol. Sci. 18, 2272. doi:10.3390/ijms18112272

Panciera, T., Azzolin, L., Cordenonsi, M., and Piccolo, S. (2017). Mechanobiology of YAP and TAZ in Physiology and Disease. Nat. Rev. Mol. Cel Biol. 18, 758-770. doi:10.1038/nrm.2017.87

Pearson, H. B., Mason, D. E., Kegelman, C. D., Zhao, L., Dawahare, J. H., Kacena, M. A., et al. (2019). Effects of Bone Morphogenetic Protein-2 on Neovascularization during Large Bone Defect Regeneration. Tissue Eng. A 25, 1623-1634. doi:10.1089/ten.tea.2018.0326

Popovic, M., Agarwal, A., Zhang, L., Yip, C., Kreder, H. J., Nousiainen, M. T., et al. (2014). Radiotherapy for the Prophylaxis of Heterotopic Ossification: a Systematic Review and Meta-Analysis of Published Data. Radiother. Oncol. 113, 10-17. doi:10.1016/j.radonc.2014.08.025

Potter, B. K., Forsberg, J. A., Davis, T. A., Evans, K. N., Hawksworth, J. S., Tadaki, D., et al. (2010). Heterotopic Ossification Following Combat-Related Trauma. J. Bone Jt. Surg Am 92 (Suppl. 2), 74-89. doi:10.2106/JBJS.J.00776

Qi, Z., Luan, J., Zhou, X., Cui, Y., and Han, J. (2017). Fibrodysplasia Ossificans Progressiva: Basic Understanding and Experimental Models. Intractable Rare Dis. Res. 6, 242-248. doi:10.5582/irdr.2017.01055

Qin, X., Zhu, B., Jiang, T., Tan, J., Wu, Z., Yuan, Z., et al. (2019). miR-17-5p Regulates Heterotopic Ossification by Targeting ANKH in Ankylosing Spondylitis. Mol. Ther. Nucleic Acids 18, 696-707. doi:10.1016/ j.omtn.2019.10.003

Qin, L., Liu, W., Cao, H., and Xiao, G. (2020). Molecular Mechanosensors in Osteocytes. Bone Res. 8, 23. doi:10.1038/s41413-020-0099-y

Ranganathan, K., Loder, S., Agarwal, S., Wong, V. W., Forsberg, J., Davis, T. A., et al. (2015). Heterotopic Ossification: Basic-Science Principles and Clinical Correlates. J. Bone Jt. Surg. 97, 1101-1111. doi:10.2106/jbjs.n.01056

Rath, E., Sherman, H., Sampson, T. G., Ben Tov, T., Maman, E., and Amar, E. (2013). The Incidence of Heterotopic Ossification in Hip Arthroscopy. Arthrosc. J. Arthrosc. Relat. Surg. 29, 427-433. doi:10.1016/j.arthro.2012.10.015

Rautela, J., Dagley, L. F., de Oliveira, C. C., Schuster, I. S., Hediyeh-Zadeh, S., Delconte, R. B., et al. (2019). Therapeutic Blockade of Activin-A Improves NK Cell Function and Antitumor Immunity. Sci. Signal. 12, eaat7527. doi:10.1126/ scisignal.aat 7527

Regard, J. B., Malhotra, D., Gvozdenovic-Jeremic, J., Josey, M., Chen, M., Weinstein, L. S., et al. (2013). Activation of Hedgehog Signaling by Loss of GNAS Causes Heterotopic Ossification. Nat. Med. 19, 1505-1512. doi:10.1038/ nm.3314

Riedl, M., Witzmann, C., Koch, M., Lang, S., Kerschbaum, M., Baumann, F., et al. (2020). Attenuation of Hypertrophy in Human MSCs via Treatment with a Retinoic Acid Receptor Inverse Agonist. Int. J. Mol. Sci. 21, 1444. doi:10.3390/ ijms 21041444

Rodgers, J. T., King, K. Y., Brett, J. O., Cromie, M. J., Charville, G. W., Maguire, K. K., et al. (2014). mTORC1 Controls the Adaptive Transition of Quiescent Stem Cells from G0 to GAlert. Nature 510, 393-396. doi:10.1038/nature13255

Rosenberg, N. M., and Bull, A. M. J. (2018). Application of a Mechanobiological Algorithm to Investigate Mechanical Mediation of Heterotopic Bone in Transfemoral Amputees. Sci. Rep. 8, 14196. doi:10.1038/s41598-018-32414-1

Rüdiger, H., Dittrich, M., Robinson, J., Mansour, T., Schwab, T., Stadelmann, V., et al. (2020). The Impact of Heterotopic Ossification on Self-Reported Outcomes after Total Hip Arthroplasty Using the Direct Anterior Approach. J. Bone Jt. Surg. Am. 102, 91. doi:10.2106/JBJS.20.00071

Rui, Y. F., Lui, P. P. Y., Ni, M., Chan, L. S., Lee, Y. W., and Chan, K. M. (2011). Mechanical Loading Increased BMP-2 Expression Which Promoted Osteogenic Differentiation of Tendon-Derived Stem Cells. J. Orthop. Res. 29, 390-396. doi:10.1002/jor.21218

Salazar, D., Golz, A., Israel, H., and Marra, G. (2014). Heterotopic Ossification of the Elbow Treated with Surgical Resection: Risk Factors, Bony Ankylosis, and
Complications. Clin. Orthop. Relat. Res. 472, 2269-2275. doi:10.1007/s11999014-3591-0

Shimono, K., Morrison, T. N., Tung, W.-e., Chandraratna, R. A., Williams, J. A., Iwamoto, M., et al. (2010). Inhibition of Ectopic Bone Formation by a Selective Retinoic Acid Receptor $\alpha$-agonist: A New Therapy for Heterotopic Ossification? J. Orthop. Res. 28, 271-277. doi:10.1002/jor.20985

Shimono, K., Tung, W.-e., Macolino, C., Chi, A. H.-T., Didizian, J. H., Mundy, C., et al. (2011). Potent Inhibition of Heterotopic Ossification by Nuclear Retinoic Acid Receptor- $\gamma$ Agonists. Nat. Med. 17, 454-460. doi:10.1038/nm.2334

Shore, E. M., and Kaplan, F. S. (2010). Inherited Human Diseases of Heterotopic Bone Formation. Nat. Rev. Rheumatol. 6, 518-527. doi:10.1038/ nrrheum.2010.122

Stanley, A., Heo, S. j., Mauck, R. L., Mourkioti, F., and Shore, E. M. (2019). Elevated BMP and Mechanical Signaling through YAP1/RhoA Poises FOP Mesenchymal Progenitors for Osteogenesis. J. Bone Miner Res. 34, 1894-1909. doi:10.1002/jbmr.3760

Suito, M., Yuzuriha, S., Iwasawa, M., Yanagisawa, D., Kinjo, Y., Takashimizu, I., et al. (2018). Therapeutic Strategies for Elbow Ankylosis Due to Heterotopic Ossification in Patients with Severe burns. JPRAS Open 17, 24-30. doi:10.1016/ j.jpra.2018.06.004

Sung Hsieh, H. H., Chung, M. T., Allen, R. M., Ranganathan, K., Habbouche, J., Cholok, D., et al. (2017). Evaluation of Salivary Cytokines for Diagnosis of Both Trauma-Induced and Genetic Heterotopic Ossification. Front. Endocrinol. 8, 74. doi:10.3389/fendo.2017.00074

Teasell, R. W., Mehta, S., Mehta, S., Aubut, J. L., Ashe, M. C., Sequeira, K., et al. (2010). A Systematic Review of the Therapeutic Interventions for Heterotopic Ossification after Spinal Cord Injury. Spinal Cord 48, 512-521. doi:10.1038/ sc.2009.175

Tu, B., Liu, S., Yu, B., Zhu, J., Ruan, H., Tang, T., et al. (2016). miR-203 Inhibits the Traumatic Heterotopic Ossification by Targeting Runx2. Cell Death Dis. 7, e2436. doi:10.1038/cddis.2016.325

Uda, Y., Azab, E., Sun, N., Shi, C., and Pajevic, P. D. (2017). Osteocyte Mechanobiology. Curr. Osteoporos. Rep. 15, 318-325. doi:10.1007/s11914017-0373-0

Vanden Bossche, L., and Vanderstraeten, G. (2005). Heterotopic Ossification: a Review. J. Rehabil. Med. 37, 129-136. doi:10.1080/16501970510027628

Vasileiadis, G. I., Sakellariou, V. I., Kelekis, A., Galanos, A., Soucacos, P. N., Papagelopoulos, P. J., et al. (2010). Prevention of Heterotopic Ossification in Cases of Hypertrophic Osteoarthritis Submitted to Total Hip Arthroplasty. Etidronate or Indomethacin? J. Musculoskelet. Neuronal. Interact 10, 159-165.

Wang, G., Kang, Y., Chen, F., and Wang, B. (2016). Cervical Intervertebral Disc Calcification Combined with Ossification of Posterior Longitudinal Ligament in An-11-Year Old Girl: Case Report and Review of Literature. Childs Nerv. Syst. 32, 381-386. doi:10.1007/s00381-015-2840-1

Wang, X., Li, F., Xie, L., Crane, J., Zhen, G., Mishina, Y., et al. (2018). Inhibition of Overactive TGF- $\beta$ Attenuates Progression of Heterotopic Ossification in Mice. Nat. Commun. 9, 551. doi:10.1038/s41467-018-02988-5

Wang, L., You, X., Lotinun, S., Zhang, L., Wu, N., and Zou, W. (2020). Mechanical Sensing Protein PIEZO1 Regulates Bone Homeostasis via Osteoblast-Osteoclast Crosstalk. Nat. Commun. 11, 282. doi:10.1038/s41467-019-14146-6

Wei, Q., Holle, A., Li, J., Posa, F., Biagioni, F., Croci, O., et al. (2020). BMP-2 Signaling and Mechanotransduction Synergize to Drive Osteogenic Differentiation via YAP/TAZ. Adv. Sci. 7, 1902931. doi:10.1002/ advs.201902931

Wong, K. R., Mychasiuk, R., O’Brien, T. J., Shultz, S. R., McDonald, S. J., and Brady, R. D. (2020). Neurological Heterotopic Ossification: Novel Mechanisms, Prognostic Biomarkers and Prophylactic Therapies. Bone Res. 8, 42. doi:10.1038/s41413-020-00119-9

Xiong, J., Almeida, M., and O'Brien, C. A. (2018). The YAP/TAZ Transcriptional Co-activators Have Opposing Effects at Different Stages of Osteoblast Differentiation. Bone 112, 1-9. doi:10.1016/j.bone.2018.04.001

Xu, J., Zeng, M., Xie, J., Wen, T., and Hu, Y. (2017). Cementless Total Hip Arthroplasty in Patients with Ankylosing Spondylitis. Medicine (Baltimore) 96, e5813. doi:10.1097/md.0000000000005813

Xu, R., Hu, J., Zhou, X., and Yang, Y. (2018). Heterotopic Ossification: Mechanistic Insights and Clinical Challenges. Bone 109, 134-142. doi:10.1016/ j.bone.2017.08.025 
Yu, K.-W., Yao, C.-C., Jeng, J.-H., Shieh, H.-Y., and Chen, Y.-J. (2018). Periostin Inhibits Mechanical Stretch-Induced Apoptosis in Osteoblastlike MG-63 Cells. J. Formos. Med. Assoc. 117, 292-300. doi:10.1016/ j.jfma.2017.12.008

Yu, T., Zhang, J., Zhu, W., Wang, X., Bai, Y., Feng, B., et al. (2021). Chondrogenesis Mediates Progression of Ankylosing Spondylitis through Heterotopic Ossification. Bone Res. 9, 19. doi:10.1038/s41413021-00140-6

Yuan, P., Wang, W.-C., Li, Z.-H., and Mao, X.-Z. (2009). Could Insertion of the Particles that Induce Osteolysis Be a New Treatment Option in Heterotopic Ossification? Med. Hypotheses 73, 27-28. doi:10.1016/j.mehy.2009.02.016

Yue, R., Zhou, B. O., Shimada, I. S., Zhao, Z., and Morrison, S. J. (2016). Leptin Receptor Promotes Adipogenesis and Reduces Osteogenesis by Regulating Mesenchymal Stromal Cells in Adult Bone Marrow. Cell Stem Cell 18, 782-796. doi:10.1016/j.stem.2016.02.015

Zaman, S. R. (2012). Heterotopic Ossification of the Elbows in a Major Petrol Burn. BMJ Case Rep. 2012. doi:10.1136/bcr.03.2012.6027

Zhang, X., Jie, S., Liu, T., and Zhang, X. (2014). Acquired Heterotopic Ossification in Hips and Knees Following Encephalitis: Case Report and Literature Review. BMC Surg. 14, 74. doi:10.1186/1471-2482-14-74

Zhang, S.-D., Xie, Z.-1., Zhang, K.-Q., Nh-Tseung, K., and Zhao, J.-J. (2018). Progressive Osseous Heteroplasia in a Chinese Infant and a Novel Mutation in the GNAS Gene. J. Eur. Acad. Dermatol. Venereol. 32, e209-e211. doi:10.1111/ jdv. 14743

Zhao, Y., Ouyang, N., Chen, L., Zhao, H., Shen, G., and Dai, J. (2020). Stimulating Factors and Origins of Precursor Cells in Traumatic Heterotopic Ossification
Around the Temporomandibular Joint in Mice. Front. Cel Dev. Biol. 8, 445. doi: $10.3389 /$ fcell.2020.00445

Zhou, X., Chadarevian, J. P., Ruiz, B., and Ying, Q.-L. (2017). Cytoplasmic and Nuclear TAZ Exert Distinct Functions in Regulating Primed Pluripotency. Stem Cel Rep. 9, 732-741. doi:10.1016/j.stemcr.2017.07.019

Zhu, S., Zhu, J., Zhen, G., Hu, Y., An, S., Li, Y., et al. (2019). Subchondral Bone Osteoclasts Induce Sensory Innervation and Osteoarthritis Pain. J. Clin. Invest. 129, 1076-1093. doi:10.1172/jci121561

Conflict of Interest: The authors declare that the research was conducted in the absence of any commercial or financial relationships that could be construed as a potential conflict of interest.

Publisher's Note: All claims expressed in this article are solely those of the authors and do not necessarily represent those of their affiliated organizations, or those of the publisher, the editors and the reviewers. Any product that may be evaluated in this article, or claim that may be made by its manufacturer, is not guaranteed or endorsed by the publisher.

Copyright $\odot 2022 \mathrm{Xu}$, Huang, He, He, Chen, Hou, Huang, Jiao, Liu, Zou, Liu and Li. This is an open-access article distributed under the terms of the Creative Commons Attribution License (CC BY). The use, distribution or reproduction in other forums is permitted, provided the original author(s) and the copyright owner(s) are credited and that the original publication in this journal is cited, in accordance with accepted academic practice. No use, distribution or reproduction is permitted which does not comply with these terms. 